\title{
Postpartum Obstetric Thromboembolism
}

National Cancer Institute

\section{Source}

National Cancer Institute. Postpartum Obstetric Thromboembolism. NCI Thesaurus.

Code C111865.

Presence of a blood clot in the maternal vascular system which orig inated from a distant site during the postpartum period. 